\title{
Intrusão dentária utilizando mini-implantes
}

\author{
Telma Martins de Araújo*, Mauro Henrique Andrade Nascimento**, \\ Fernanda Catharino Menezes Franco ${ }^{\star \star \star}$, Marcos Alan Vieira Bittencourt ${ }^{\star \star \star \star}$
}

\section{Resumo}

Introdução: dentre os diversos tipos de movimentos dentários induzidos ortodonticamente, o de intrusão é, sem dúvida, um dos mais difíceis de serem conseguidos. A mecânica intrusiva convencional, apesar de viável, é complexa, no que diz respeito ao controle de seus efeitos colaterais. Isso, em grande parte, refere-se à dificuldade em se obter uma ancoragem satisfatória. Neste contexto, os mini-implantes, por oferecerem efetiva ancoragem esquelética, têm se mostrado de extrema valia para os ortodontistas, tornando a intrusão, tanto de dentes anteriores quanto posteriores, um procedimento cada vez mais simples, do ponto de vista mecânico. Objetivo: pretende-se, então, neste artigo, descrever e demonstrar, clinicamente, as diversas possibilidades de utilização dos mini-implantes como recurso de ancoragem para o movimento de intrusão.

Palavras-chave: Mini-implante. Intrusão dentária. Ancoragem esquelética.

\section{INTRODUÇÃO}

Em muitos tratamentos ortodônticos, um adequado planejamento da ancoragem é imprescindível para o sucesso da terapia proposta. A intrusão dentária, seja para a correção da sobremordida exagerada ou da mordida aberta anterior, seja para a correção de dentes extruídos pela falta dos antagonistas, representa grande desafio mecânico, pela dificuldade de controle de movimentos indesejáveis nas unidades de ancoragem. Obviamente, ao longo dos anos, a literatura tem apontado resultados satisfatórios com a utilização de aparelhos auxiliares extra e intrabucais. Porém, nem sempre é fácil conseguir do paciente a colaboração necessária, pelo desconforto físico e/ou estético que esses aparelhos podem causar.

Neste contexto, excelente alternativa é a utili- zação dos mini-implantes. Seu desenvolvimento, ao longo dos últimos anos, tem proporcionado uma ancoragem bastante eficiente, que prescinde da utilização de dentes e não acarreta qualquer comprometimento estético, além de demandar mínima ou nenhuma colaboração do paciente ${ }^{1,2}$. Este recurso tem sido utilizado cada vez mais freqüentemente na clínica ortodôntica, quando não há unidades dentárias suficientes para promover efetiva ancoragem ou, simplesmente, de modo a tornar a mecânica menos complexa e mais previsível para o ortodontista ${ }^{1}$.

Pretende-se, neste artigo, sumarizar e exemplificar diversas possibilidades de utilização dos miniimplantes, com vistas à intrusão dentária, destacando-se, em algumas situações, cuidados que devem ser adotados para se obter os efeitos almejados.

\footnotetext{
* Doutora e Mestre em Ortodontia pela UFRJ. Professora Titular de Ortodontia da UFBA. Coordenadora do Curso de Especialização em Ortodontia da UFBA. Diretora do Board Brasileiro de Ortodontia e Ortopedia Facial.

** Aluno do Curso de Especialização em Ortodontia da UFBA

*** Mestre em Ortodontia pela UFRJ. Professora Assistente de Ortodontia da FBDC.

**** Doutor e Mestre em Ortodontia pela UFRJ. Professor Adjunto de Ortodontia da UFBA. Diplomado pelo Board Brasileiro de Ortodontia e Ortopedia Facial.
} 


\section{INTRUSÃO DE INCISIVOS}

A intrusão de dentes anteriores, indicada em alguns casos de sobremordida exagerada, tem sido realizada, tradicionalmente, por meio de arcos de intrusão com degraus (na região anterior), com curva acentuada (no arco superior) ou curva reversa (no arco inferior). Em muitas situações, porém, os efeitos colaterais destas mecânicas são inevitáveis, especialmente a extrusão ou a inclinação das unidades de ancoragem. Com a ancoragem esquelética obtida por meio dos mini-implantes, os demais dentes ficam resguardados de qualquer movimento indesejado.

A posição ideal para a instalação dos miniimplantes com a finalidade de intruir os incisivos superiores depende da inclinação destes. Quando se apresentam verticalmente dispostos ou retroinclinados, como na Classe II, $2^{a}$ divisão de Angle, recomenda-se ${ }^{8}$ utilizar um único mini-implante na linha média, o mais alto possivel, próximo à espinha nasal anterior. Para a intrusão dos incisivos inferiores, também verticalizados ou retroinclinados, o mini-implante deve ser posicionado, entre os centrais, o mais baixo possível ${ }^{6,8,11}$. Nesta posição, a linha de força passará bem à frente do centro de resistência do conjunto, gerando um efeito de intrusão associado à inclinação vestibular destas unidades (Fig. 1).

Quando os incisivos se encontram com boa inclinação axial e, portanto, não se deseja alterála, deve-se fazer com que a linha de ação de força passe o mais próximo possível do centro de resistência do conjunto de dentes que serão movimentados $^{6,11}$. Para isto, sugere-se utilizar dois mini-implantes, um de cada lado, posicionados entre os incisivos laterais e os caninos (Fig. 2). Um exemplo clínico da utilização de dois miniimplantes com este objetivo pode ser visualizado na figura 3. Neste caso, como o paciente possuía apenas três incisivos inferiores, optou-se pela re-
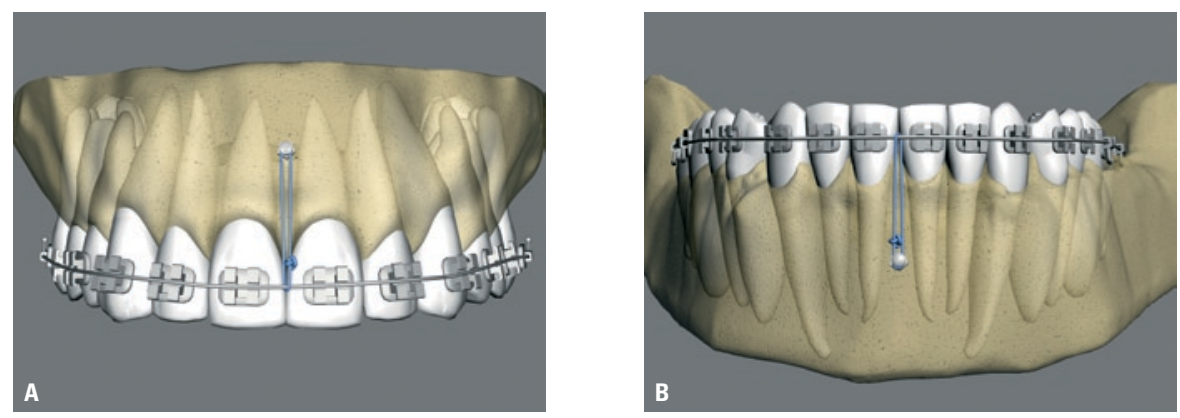

FIGURA 1 - Intrusão de incisivos superiores e inferiores, quando é desejável a inclinação para vestibular destes dentes.
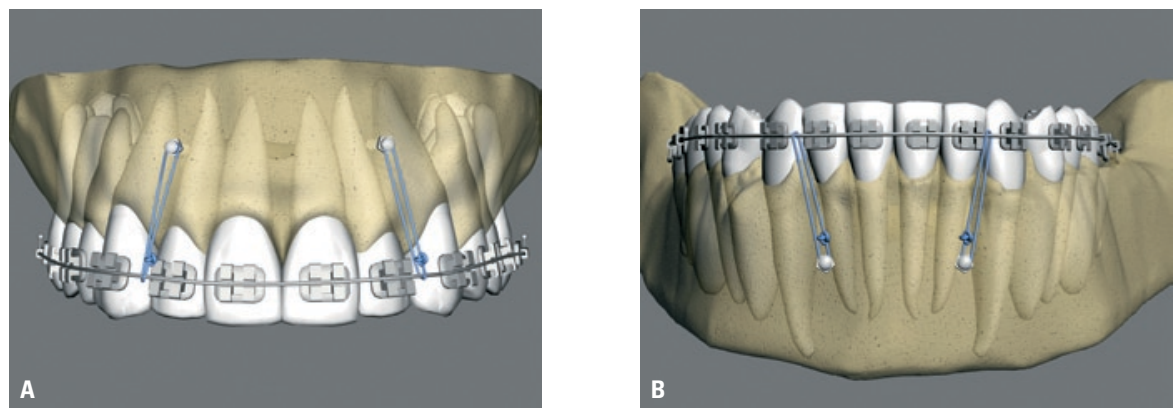

FIGURA 2 - Intrusão de incisivos superiores e inferiores, quando é desejável manter a inclinação axial destes dentes. 
moção de um deles e migração mesial dos caninos, que passaram a ocupar a posição dos incisivos laterais. Este conjunto de dentes já se encontrava inclinado para vestibular $\left(\mathrm{IMPA}=109^{\circ}\right)$. Assim, desejava-se que a intrusão fosse realizada, objetivando-se o nivelamento da curva de Spee, sem que esta inclinação fosse agravada. Como pode ser visto, o movimento de intrusão ocorreu e a inclinação vestíbulo-lingual dos incisivos inferiores mostrou-se, ao final do movimento, até discretamente melhorada $\left(\mathrm{IMPA}=107^{\circ}\right)$.
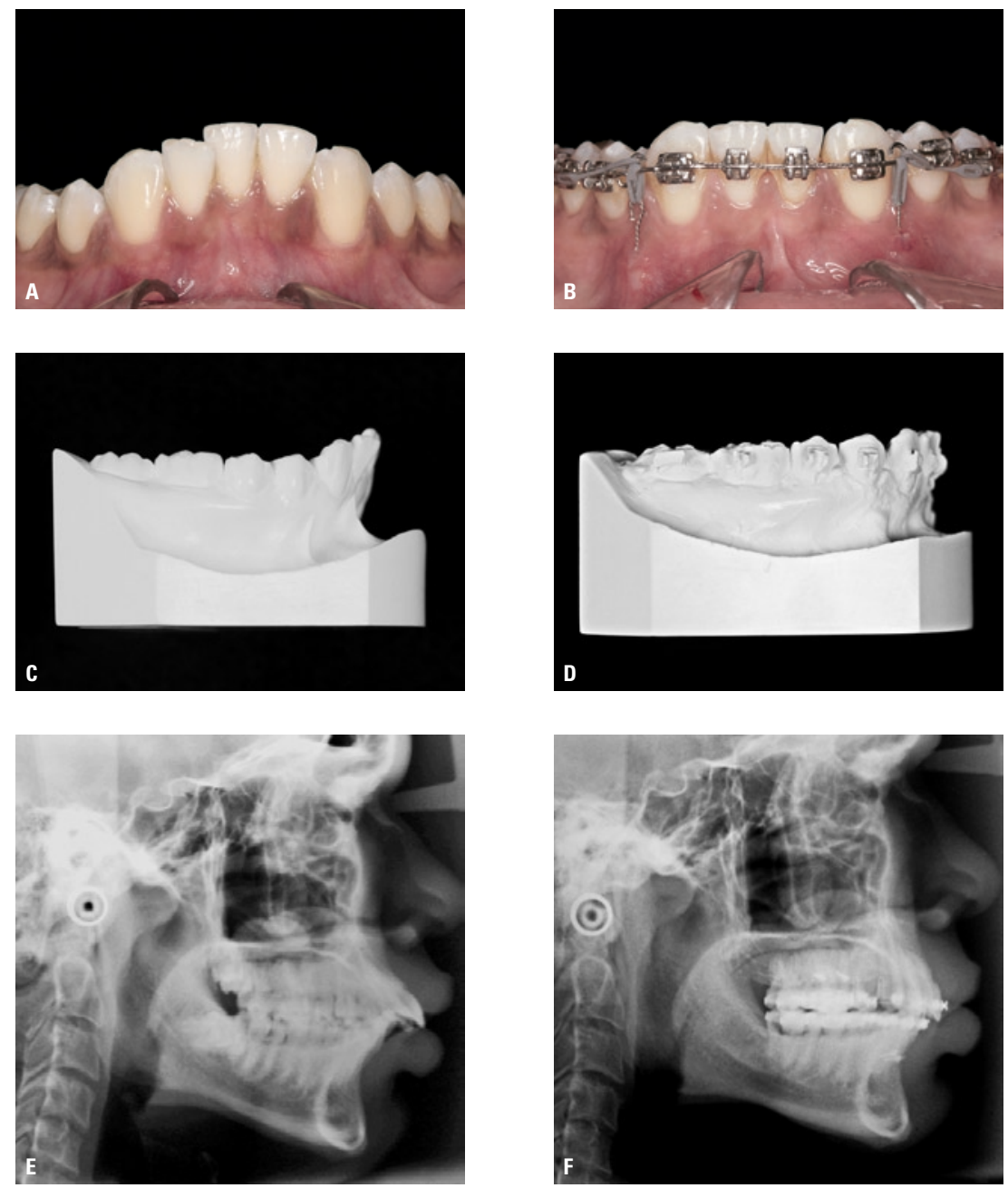

FIGURA 3 - Intrusão de unidades ântero-inferiores, com mini-implantes submersos instalados em mucosa alveolar (A, B). Em C e D, observa-se o nivelamento da curva de Spee. Pode-se visualizar, em E e F, a discreta melhora na inclinação vestíbulo-lingual das unidades intruídas. 
nos incisivos e permite a realização de dobras de compensação.

À medida que a intrusão acontece, é importante checar, em vista anterior, a forma do arco e o plano oclusal, que podem sofrer modificações caso a intrusão não ocorra simetricamente nos hemiarcos direito e esquerdo. Outro fator que deve ser monitorado é o torque dos dentes anteriores, muitas vezes perdido quando a intrusão é realizada com arcos leves ${ }^{14}$.

\section{INTRUSÃO DE CANINOS}

$\mathrm{Na}$ mecânica convencional, os caninos são intruídos, geralmente, por meio de arcos contínuos, com dobras de segunda ordem ou tipo by pass, associadas a elásticos, utilizando-se os dentes vizinhos como ancoragem. Nestes casos, o componente extrusivo nas unidades de ancoragem é inevitável. Outra alternativa é o emprego de arcos segmentados, tendo-se como ancoragem os dentes posteriores. Quando o paciente apresenta perdas dentárias nesta área, ou comprometimento periodontal nos dentes presentes, este tipo de mecânica se torna inviável.

Com a utilização dos mini-implantes, os efeitos indesejáveis e/ou limitações deixam de ocorrer. Quando se deseja a intrusão do canino, mantendose sua inclinação axial, sugere-se a utilização de dois mini-implantes por vestibular, um na mesial e outro na distal. Este cuidado é importante por- que a utilização de apenas um mini-implante irá gerar, além da força intrusiva, um componente de força para distal ou mesial, a depender da posição do mesmo, que tende a inclinar o dente. Neste exemplo, a ativação apenas por vestibular promoverá um componente de torque palatino de raiz, à medida que o movimento de intrusão do canino ocorre. Para controlar este efeito indesejado, podese confeccionar um arco de aço inoxidável 0,019" x 0,026 " contínuo, tangenciando a face vestibular do canino, logo abaixo do braquete. É importante enfatizar que o contato do fio com a superfície dentária é essencial para o controle deste efeito e, portanto, deve ser checado e ajustado, se necessário, a cada consulta ${ }^{1}$ (Fig. 4).

Uma alternativa que pode ser utilizada é a colocação de um mini-implante por vestibular, na mesial do canino, e outro por palatino, em sua face distal, ou vice-versa, ativando-se o conjunto com a colocação de um elástico que une um miniimplante ao outro, passando pelo centro da coroa deste dente. Freqüentemente, para que o elástico permaneça estável em sua posição, é necessária a colocação de uma ponte de resina composta, estrategicamente posicionada na coroa do canino.

\section{INTRUSÃO DE DENTES POSTERIORES}

A necessidade de intrusão dos dentes posteriores ocorre, principalmente, em função da perda de unidade(s) antagonista(s) ou quando
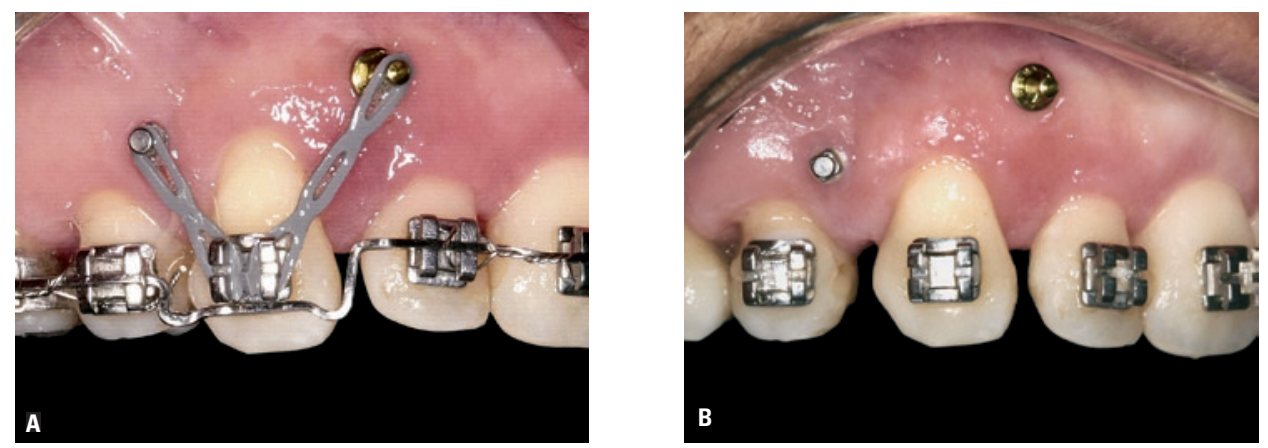

FIGURA 4 - Intrusão de canino superior com arco 0,019" x 0,026" tangenciando a unidade, para evitar sua inclinação para vestibular. 
há excesso vertical na região posterior, causando mordida aberta anterior ${ }^{3}$. Quando comparada à intrusão de dentes anteriores, a intrusão na região posterior é um movimento mais difícil de ser obtido, devido ao maior volume radicular dos molares e pré-molares, o que proporciona maior reação do osso alveolar e maior tempo de tratamento. $\mathrm{O}$ controle tridimensional da posição dos dentes é fundamental para o sucesso da intrusão posterior. Além da posição vertical, a forma do arco, a inclinação axial dos dentes, a inclinação do plano oclusal e o torque posterior devem ser planejados de acordo com os objetivos individuais do tratamento ${ }^{14}$. A maioria dos casos requer movimento dentário em corpo e, portanto, algumas dificuldades devem ser consideradas, como a localização do centro de resistência, que sofre influência de diferenças individuais, a forma da raiz e o nível do tecido ósseo, além de condições anatômicas que, muitas vezes, impedem a instalação dos mini-implantes na posição ideal ${ }^{7,14,21}$.

\section{Intrusão de uma unidade}

Diante da perda de unidades dentárias na região posterior, tem-se, freqüentemente, a extrusão de um ou mais dentes no arco antagonista. Esta extrusão, além de comprometer o espaço para a reabilitação protética, pode causar outros transtornos, como defeitos periodontais e interferências oclusais durante os movimentos funcionais ${ }^{25}$. Assim, é importante a correção deste problema, promovendo-se a intrusão do dente envolvido.

No arco superior, se apenas um dente posterior necessitar de intrusão, deve-se instalar dois miniimplantes, um por vestibular e outro por palatino, sendo um na mesial e o outro na distal. Os miniimplantes assim dispostos proporcionam um movimento vertical controlado, sem inclinações indesejáveis ${ }^{25}$. A aplicação da força pode ser feita tanto acoplando elásticos partindo dos mini-implantes e indo até acessórios ortodônticos instalados nas faces vestibular e palatina do dente em questão (Fig. 5A), quanto passando o elástico diretamente por sobre a superfície oclusal do mesmo, ligando um mini-implante ao outro (Fig. 5B). Nesse caso, deve-se ter atenção com a linha de ação de força, de modo que não ocorra deslocamento do elástico para mesial ou distal, o que poderia levar à inclinação da unidade dentária que está sendo intruí$\mathrm{da}^{1,2,16}$.

\section{Intrusão de grupo de dentes}

Antes do advento dos mini-implantes, as principais alternativas para a reabilitação de um paciente que apresentava um grupo de dentes extruídos na região posterior eram, na maioria dos casos, desgastes na superfície oclusal destas unidades ou um tratamento cirúrgico combinado, com a impacção desta região $0^{5,17,23}$.

Atualmente, com o auxílio da ancoragem esquelética, pode-se realizar a intrusão ortodôntica controlada destas unidades. Em se tratando de di-
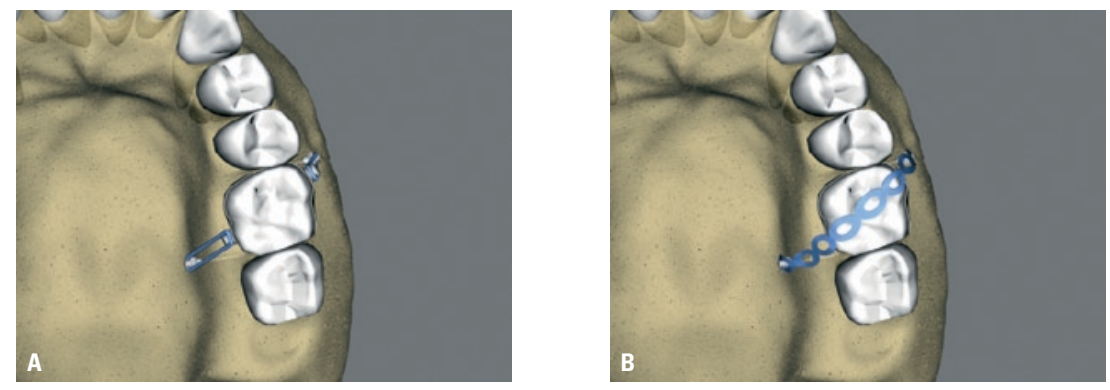

FIGURA 5 - Mini-implantes para intrusão do primeiro molar superior, ativados com elástico em fio, por vestibular e palatino (A), e com elástico em cadeia, passando pela superfície oclusal (B). 

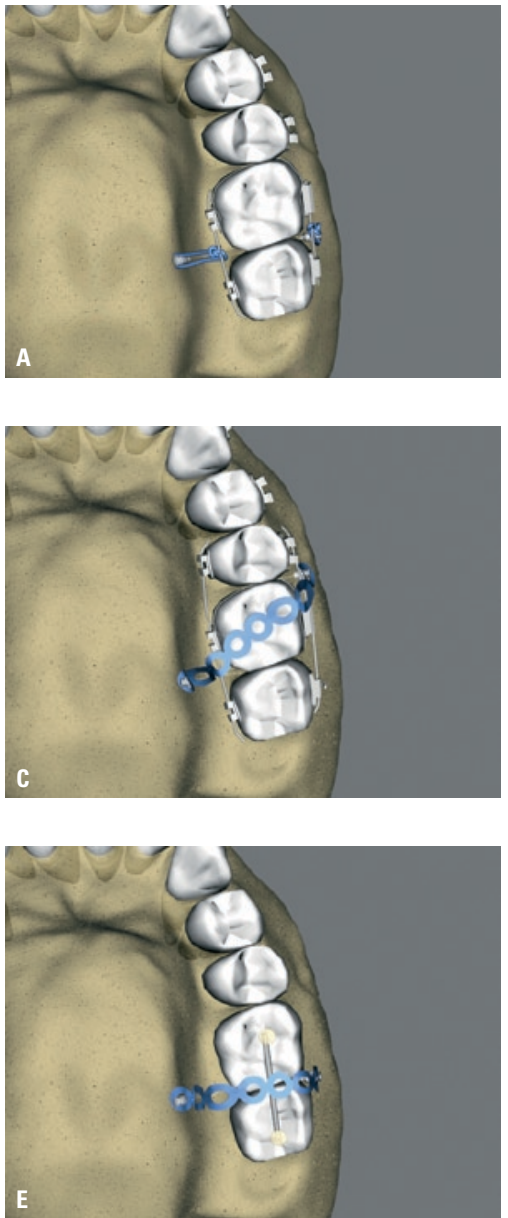
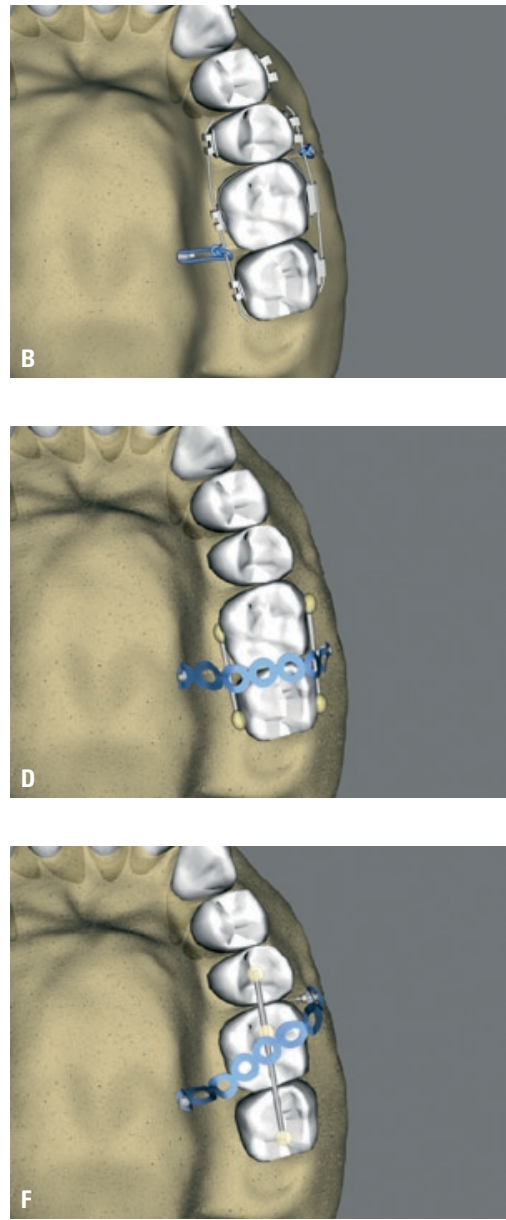

FIGURA 6 - Diferentes formas de intrusão de um grupo de dentes posteriores, com segmentos de fio fixados a braquetes, por vestibular e palatino (A, B, C), colados diretamente nestas superfícies (D) ou fixados sobre a superfície oclusal (E, F). Observa-se que a ativação pode ser realizada com elástico em fio acoplados aos segmentos de $\operatorname{arcos}(\mathbf{A}, \mathbf{B})$ ou com elástico em cadeia, passando sobre a superfície oclusal (C a F).

versos dentes a serem intruídos, estes devem ser unidos em bloco ${ }^{1,2,4}$. Pode-se instalar braquetes nas superfícies vestibular e palatina dos mesmos e uni-los com arcos segmentados, pode-se colar diretamente um fio ortodôntico, na vestibular e/ou palatina ou, ainda, fixar um único segmento de fio ortodôntico passando pelas superfícies oclusais, desde que não cause interferência (Fig. 6).

Mesmo para um número maior de dentes, em geral, dois mini-implantes suportam bem a carga ${ }^{2,3}$. Como pode ser visto na figura 7A, a perda de dentes no segmento posterior direito do arco inferior determinou a extrusão do segundo pré-molar e dos primeiro e segundo molares superiores. Como o primeiro molar se encontrava mais extruído que os demais, os dois mini-implantes instalados foram utilizados, inicialmente, para promover sua intrusão (Fig. 7B), até que os dentes do hemiarco direito estivessem nivelados. Então, o pré-molar e os molares foram unidos com um fio colado na superfície oclusal dos mesmos e o sistema voltou a ser ativado com elástico, para intrusão em bloco (Fig. 7C, D). Na figura 7E, pode-se visualizar o resultado obtido. Outro exemplo de utilização de mini-implantes com esta mesma finalidade pode ser visualizado na figura 8 . 

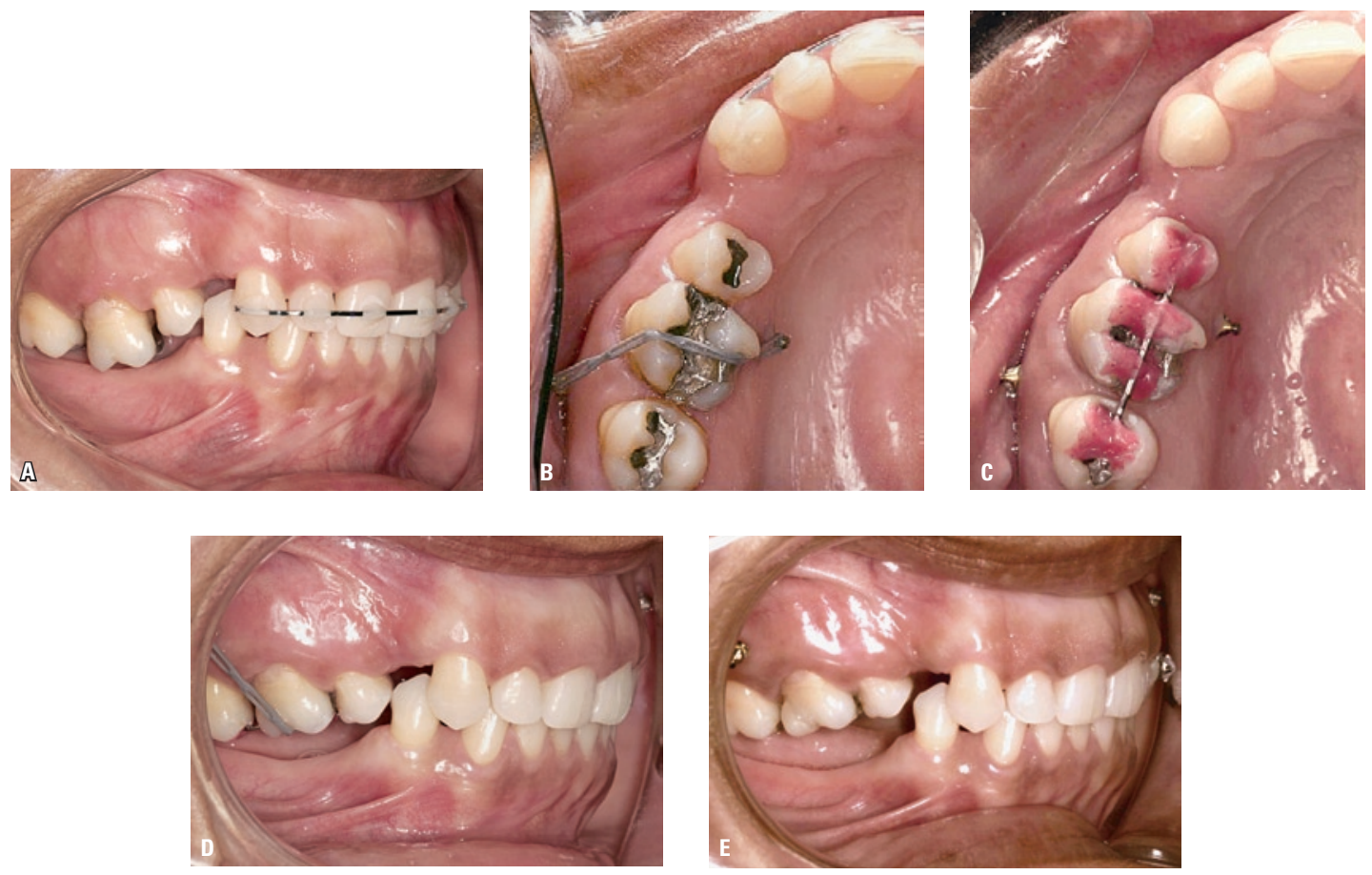

FIGURA 7 - Caso clínico apresentando extrusão dos dentes posteriores superiores, devido à perda dos elementos antagonistas (A). Em B, ativação para intrusão do primeiro molar, que se encontrava mais extruído, utilizando dois mini-implantes. Observa-se o aumento, com resina, da cúspide mesiopalatina, com o objetivo de guiar o posicionamento do elástico em cadeia, impedindo seu deslocamento para mesial, o que poderia resultar na inclinação deste dente. 0 pré-molar e os molares foram unidos com um fio colado na superfície oclusal (C) e o sistema voltou a ser ativado com elástico para intrusão em bloco (D). Em E, pode-se visualizar o movimento obtido.

\section{Correção da mordida aberta anterior}

A mordida aberta anterior, especialmente em pacientes adultos, é uma alteração que apresenta grande dificuldade de correção e contenção ${ }^{8,9,10}$. Do ponto de vista dentário, sua etiologia pode estar relacionada à deficiência de crescimento alveolar na região anterior, ao excesso de crescimento alveolar na região posterior ou ambos. Durante o desenvolvimento da dentição, estes problemas, em geral, são facilmente contornáveis. Contudo, à medida que cessa a fase de crescimento, torna-se cada vez mais difícil sua correção através dos métodos convencionais.

Quando o planejamento envolve a intrusão dos dentes posteriores, os mini-implantes aparecem, mais uma vez, como excelente opção de ancoragem. No caso exemplificado na figura 9, foi necessária a intrusão em ambos os lados. Assim, utilizou-se um mini-implante por vestibular e outro por palatino, nos lados direito e esquerdo. Como os dentes, na região posterior, apresentavam correto nivelamento, a força para a intrusão foi aplicada em arcos contínuos. Em algumas situações, é recomendável a fixação de um segmento de arco nas faces palatinas dos dentes, para evitar o contato dos elásticos ou molas, utilizados para a intrusão, com a mucosa do palato.

Outra alternativa é a instalação de mini-implantes apenas por vestibular. Neste caso, para o controle do torque dos dentes que serão intruídos, sugere-se utilizar, na maxila, uma barra transpalatina afastada do palato na mesma quantidade de milímetros planejada para a intrusão e, na mandíbula, uma barra lingual afastada dos incisivos ${ }^{12,19,24}$ (Fig. 10). Caso haja uma deficiência transversa associada, pode-se manter o aparelho utilizado para 

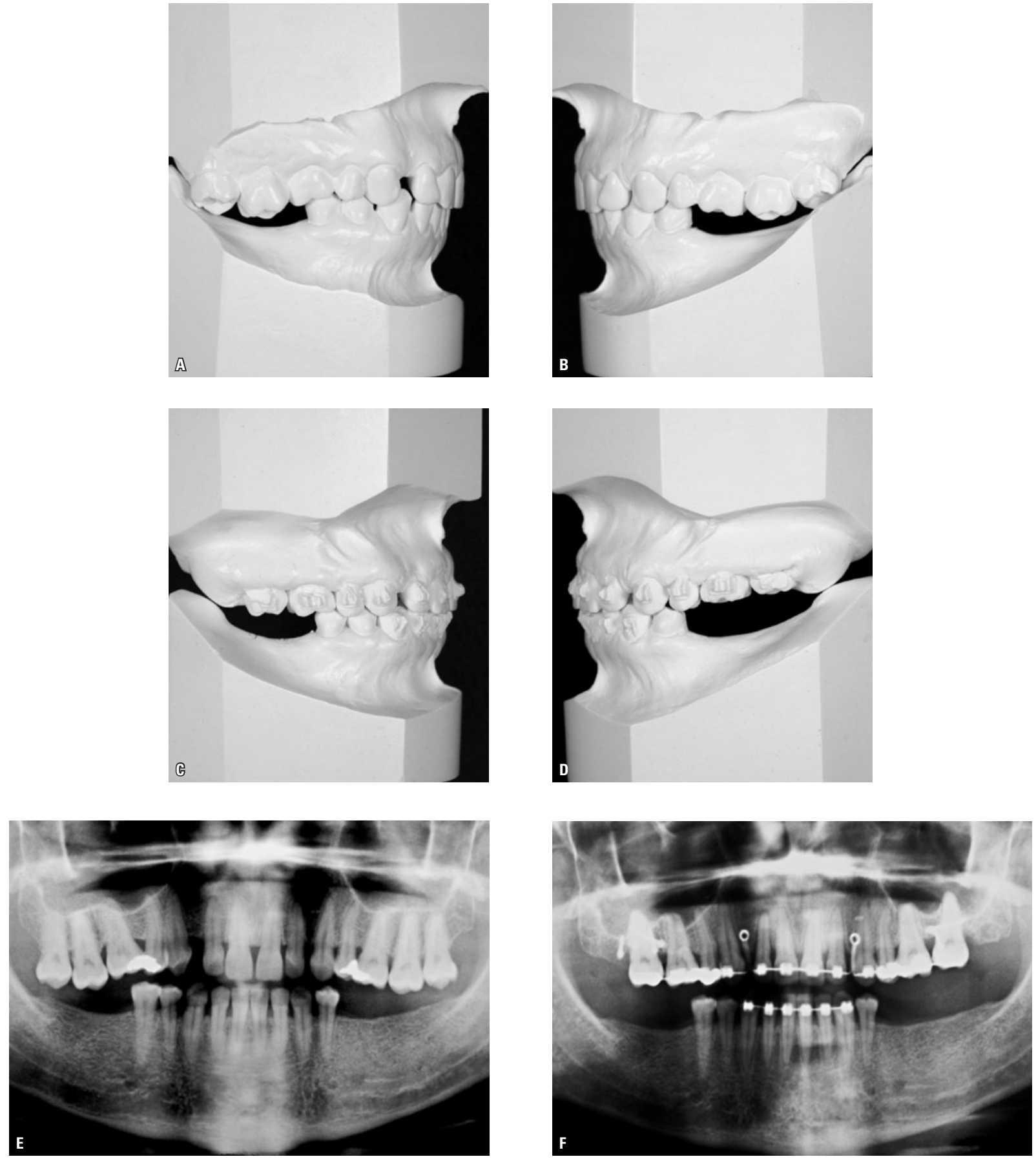

FIGURA 8 - Intrusão das unidades posteriores no arco superior, para permitir a reabilitação com implantes no arco inferior. A melhora obtida pode ser visualizada na comparação entre os modelos e radiografias iniciais (A, B, E) e após a intrusão dos molares (C, D, F). 

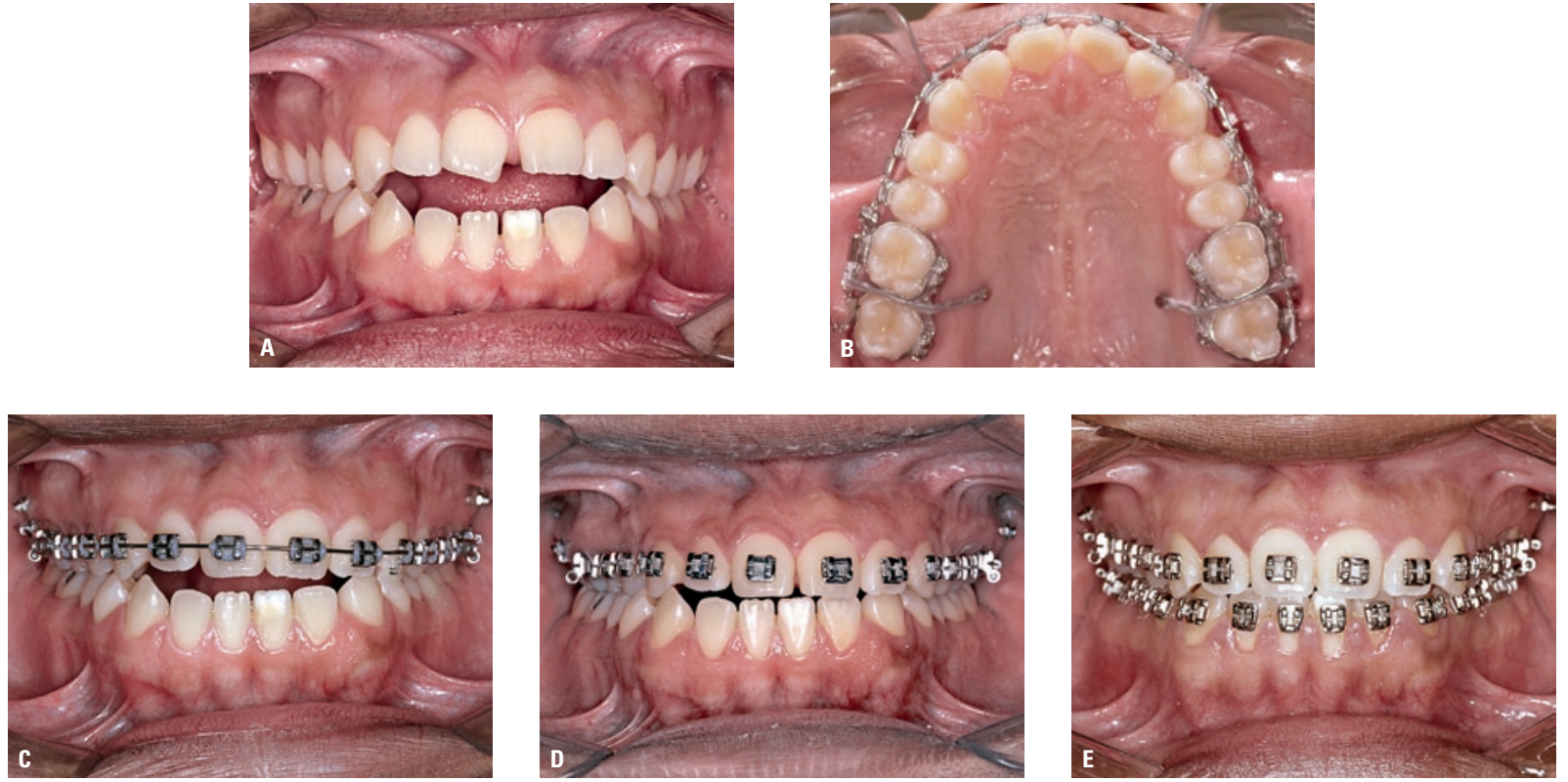

FIGURA 9 - Correção da mordida aberta anterior, com a intrusão dos segmentos posteriores do arco superior. Este movimento foi obtido por meio da utilização de miniimplantes instalados nas superfícies vestibulares e palatinas, entre os primeiros e segundos molares. Em E, observa-se o estágio atual.
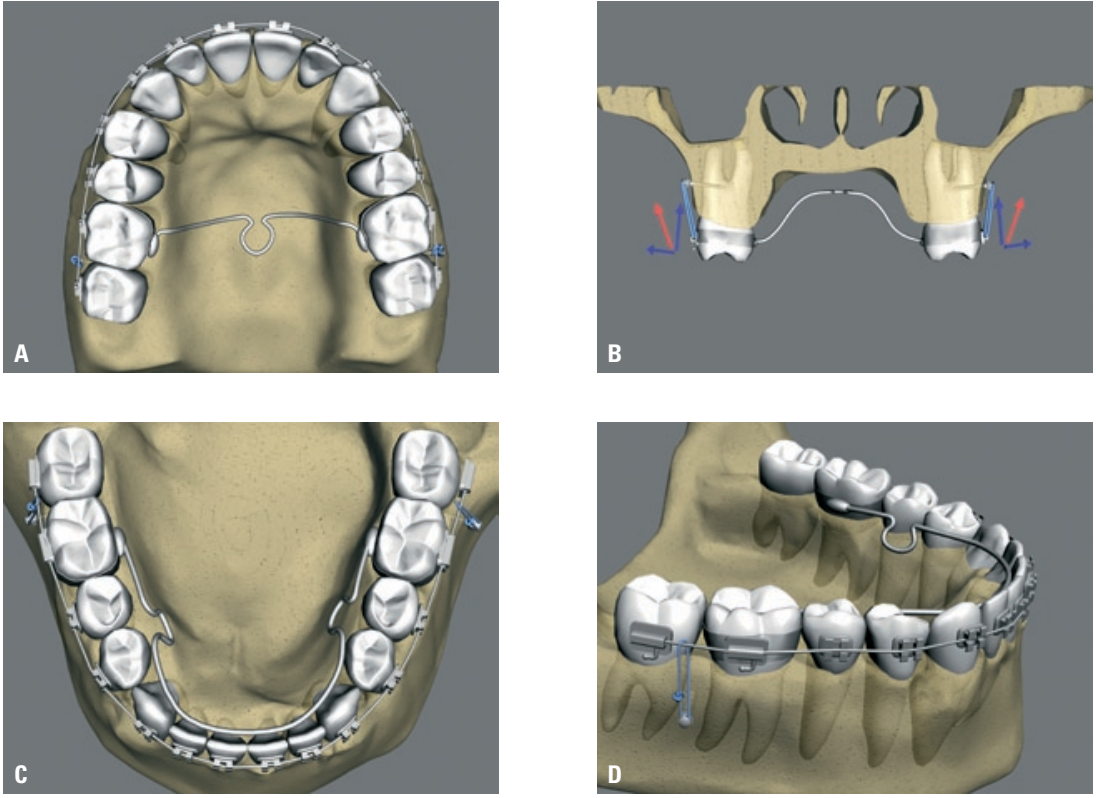

FIGURA 10 - Intrusão dos dentes posteriores, com o uso de mini-implantes apenas por vestibular. Para evitar a inclinação desses dentes, na direção de aplicação da força, devem ser instalados, no arco superior, uma barra transpalatina afastada do palato (A, B) e, no arco inferior, uma barra lingual afastada dos incisivos, cujo controle pode ser realizado por meio de alças verticais (C, D). 
a expansão superior, como pode ser visto na figura 11. Neste caso, optou-se pela utilização do Hyrax, que foi confeccionado distante do palato, na mesma magnitude almejada para a intrusão.

Outro detalhe que requer atenção é a relação incisal ântero-posterior. Se a sobressaliência inicial for pequena, pode ocorrer trauma nos incisivos quando do fechamento da mordida, em função do giro da mandíbula no sentido anti-horário. Assim, para evitar este problema, devem-se retrair, inicialmente, os dentes inferiores ou projetar os superiores, criando, desta forma, a sobressaliência necessária ${ }^{19}$.

Com o objetivo de evitar recidiva, uma força extrabucal de tração alta pode ser recomendada para uso noturno. É importante, também, acompanhamento fonoaudiológico, para que uma postura correta da língua esteja assegurada, evitandose problemas futuros, por conta de alterações na posição dos incisivos ${ }^{19,20}$.

\section{CORREÇÃO DO PLANO OCLUSAL}

Em casos de inclinação do plano oclusal, numa vista frontal, tanto na região anterior quanto na região posterior, a instalação de mini-implantes em locais estratégicos permite a utilização de força de diferente magnitude em um dos lados, promovendo a correção deste desvio. Isto se aplica para ambos os arcos, superior e inferior ${ }^{24}$. Um exemplo da utilização deste recurso pode ser visto na figura 12. A paciente apresentava, numa vista frontal, grande diferença entre os lados direito e esquerdo, com o lado direito flagrantemente mais baixo. Foi instalado, então, um mini-implante entre o canino e o primeiro pré-molar, com ativação diretamente no arco contínuo da aparatologia ortodôntica.

Inclinações oclusais mais severas podem ser encontradas em pacientes com perda de unidades dentárias, portadores de assimetrias faciais ou disfunções musculares graves e em algumas patologias localizadas. Esse problema é de difícil solução
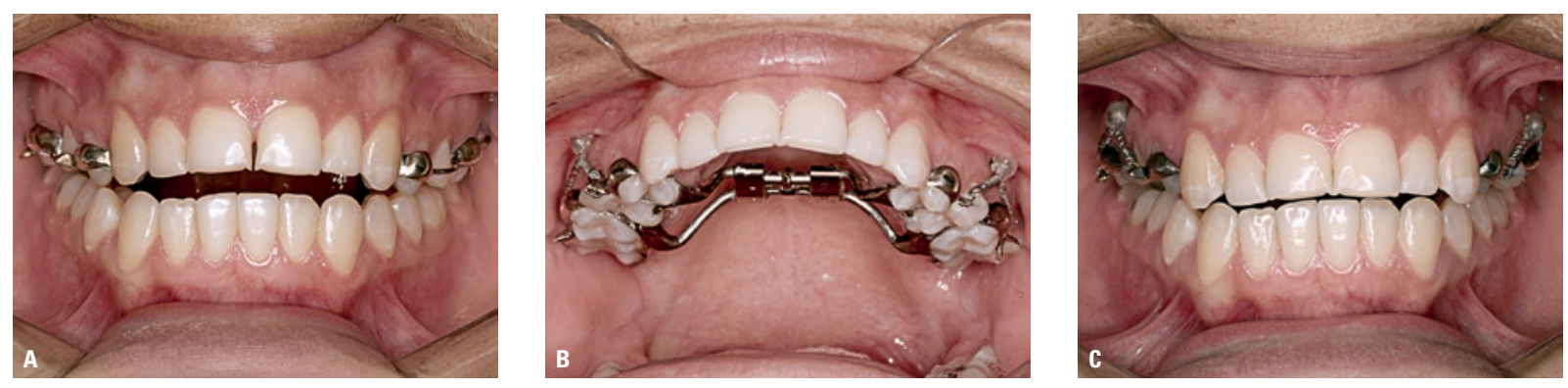

FIGURA 11 - Intrusão dos dentes posteriores, com o uso de mini-implantes apenas por vestibular. 0 aparelho de Hyrax, utilizado para a correção do problema transverso, proporcionou, durante o movimento, o controle da inclinação no sentido vestíbulo-lingual. Vale ressaltar que os segundos pré-molares e os segundos molares também foram incluídos no conjunto, de forma que todos os dentes posteriores fossem intruídos.
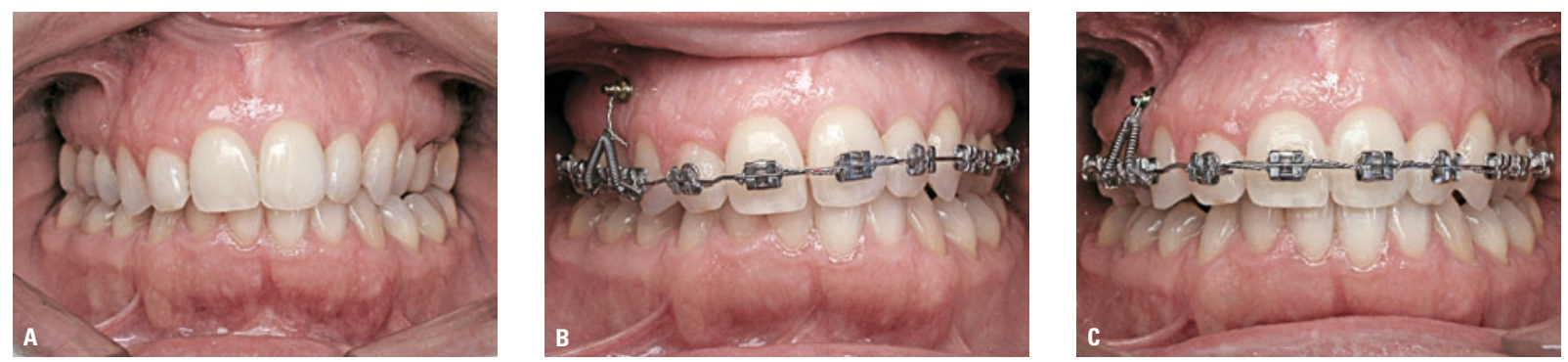

FIGURA 12 - Aplicação de um mini-implante, apenas no lado direito do arco superior, para a intrusão deste segmento e correção do plano oclusal. 
por meio dos recursos ortodônticos convencionais. A utilização de mini-implantes, nestes casos, torna a mecânica de intrusão do segmento do arco que está desnivelado bem mais simples ${ }^{6}$.

\section{CONSIDERAÇÕES GERAIS}

Conforme já mencionado, os mini-implantes instalados com o objetivo de ancorar o movimento de intrusão devem ser posicionados o mais apicalmente possível, tanto no arco superior quanto no inferior, respeitando-se, em geral, os limites da mucosa ceratinizada. Este distanciamento proporciona maior possibilidade de ativação do sistema, além de diminuir, durante a intrusão, o risco de lesões às raízes das unidades dentárias adjacentes, que ocorreriam pela proximidade com uma área mais ampla da superfície radicular ${ }^{21}$. Deve-se evitar, contudo, a região de mucosa alveolar, pois, nesta região, o risco de inflamação local é maior, o que compromete a estabilidade do mini-implante ou favorece seu recobrimento pelos tecidos moles. Neste contexto, alguns autores ${ }^{15,19}$ relatam que, na região posterior, quanto mais apical estiver o miniimplante, mais perpendicular à cortical óssea ele deve ser posicionado, de forma a evitar a perfuração do seio maxilar ${ }^{21}$.

Em alguns casos, porém, quando o paciente apresenta uma faixa muito estreita de mucosa ceratinizada, é necessária a instalação na região de mucosa alveolar. Sendo assim, uma alternativa é instalar o mini-implante submerso, com um fio de amarrilho proporcionando ligação com o meio externo, de forma a tornar possível a ativação com molas ou elásticos (Fig. 3B). É necessária uma incisão para dar acesso à penetração da fresa em forma de lança ou da broca helicoidal, o que for indicado, de acordo com a densidade óssea. No momento da instalação, deve-se distender a mucosa alveolar e afastar levemente os limites do retalho para que o tecido mole não enrosque nas espiras do mini-implante. Após a instalação e colocação do amarrilho, o retalho deve ser fechado com um ou dois pontos de sutura.
Como já pôde ser observado, a quantidade e o local de instalação dos mini-implantes estão na dependência direta da quantidade e da região dos dentes a serem intruídos. Geralmente, são necessários dois mini-implantes, um por vestibular e outro por palatino, estrategicamente colocados, na região onde se deseja atuar. Desta forma, consegue-se a intrusão do dente ou segmento em questão, controlando-se, ao mesmo tempo, a inclinação vestíbulo-palatina. Vale ressaltar que, no arco inferior, a colocação do mini-implante no lado lingual, embora desejável para o controle do torque, causa bastante desconforto ao paciente. Neste caso, além da barra lingual anteriormente citada (Fig. 10C, D), outra alternativa é controlar os efeitos colaterais com a utilização de um arco contínuo de aço inoxidável, bastante rígido $(0,0215$ ” x 0,027 ”, por exemplo), acentuando-se o torque vestibular de raiz nos dentes a serem intruídos. Em se tratando de apenas um dente, pode-se utilizar o contato do fio ortodôntico com a superfície vestibular da unidade em questão, logo acima do braquete, de forma semelhante à exemplificada no canino superior (Fig. 4).

Quando se tem como objetivo a intrusão de maior número de dentes, pode-se utilizar maior quantidade de mini-implantes (Fig. 13). Contudo, deve-se ter em mente ${ }^{13}$ que cada mini-implante suporta uma carga de até $450 \mathrm{cN}$ e que a força ótima para a movimentação ortodôntica deve ser aquela que estimula a atividade celular sem ocluir completamente os vasos sangüíneos. Para efeito de exemplificação, a força ideal ${ }^{20,22}$ para a intrusão de um molar superior é de, aproximadamente, $150 \mathrm{cN}$. Assim, na maioria dos casos, poucos mini-implantes são suficientes para a realização do movimento de intrusão, embora seja extremamente relevante considerar o sistema empregado, as condições do osso alveolar de suporte e a resposta individual do paciente. Vale ressaltar que o movimento intrusivo, por demandar maior área de reabsorção óssea, ocorre, em média, mais lentamente que outros movimentos ortodônticos. Em alguns casos, 

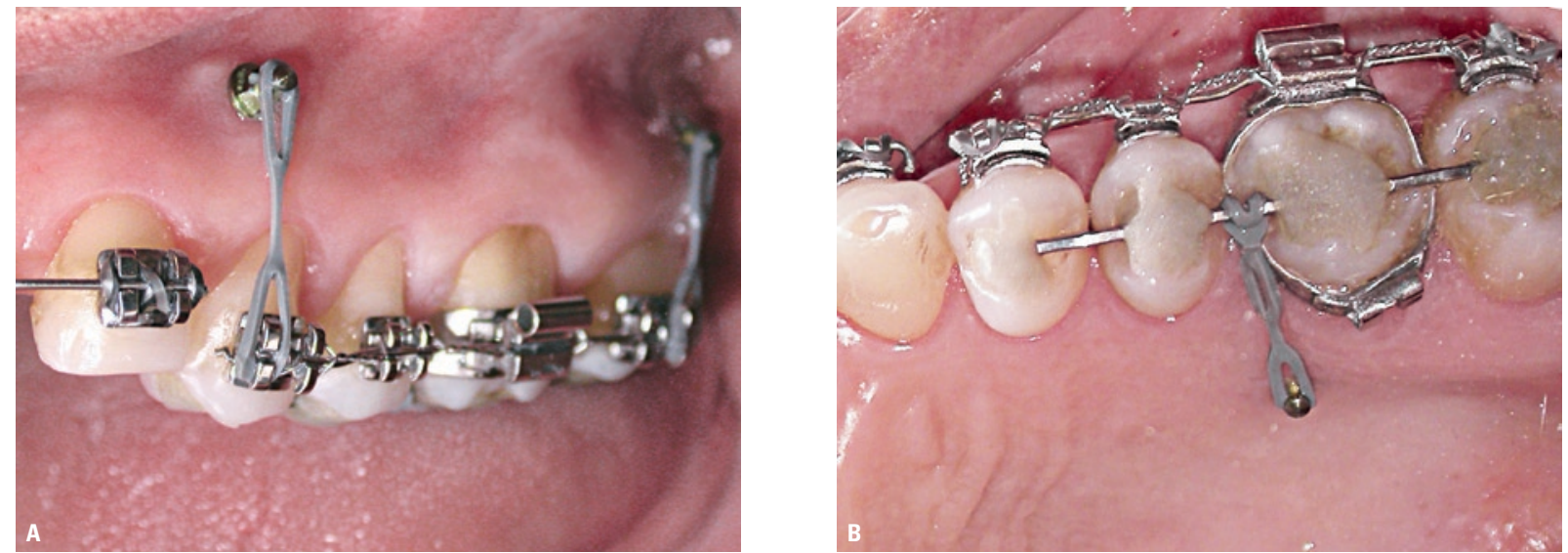

FIGURA 13 - Intrusão de quatro dentes posteriores, com a utilização de dois mini-implantes por vestibular. Como não havia dentes antagonistas, o controle da inclinação no sentido vestíbulo-lingual foi realizado com um segmento de fio colado na superfície oclusal destas unidades, acoplado a um mini-implante instalado por palatino.

observa-se um período de até três meses de inércia antes que qualquer alteração na posição dentária seja notada. Deve-se, portanto, aguardar o início da movimentação, sem aumentar a intensidade da força aplicada, pois, em geral, uma vez rompida a inércia, a intrusão tem início e se mantém, com certa constância, a uma taxa de, aproximadamente, $0,3 \mathrm{~mm}$ ao mês.

Um importante aspecto que deve ser avaliado, previamente à decisão de se intruir um determinado dente, por meio de radiografias periapicais e/ ou interproximais, é o nível ósseo entre este e os dentes adjacentes. Segundo Mathews e Kokich ${ }^{18}$, caso este acompanhe o mesmo desnível observado nas cristas marginais dos dentes envolvidos, o nivelamento das cristas através da intrusão também nivelará o osso. Entretanto, se o nível ósseo entre os dentes adjacentes for plano, a correção ortodôntica desta discrepância, através da intrusão, produzirá um defeito ósseo vertical e, conseqüentemente, bolsa periodontal na face proximal da unidade. Nesta situação, de acordo com os autores, a melhor conduta seria o nivelamento do plano oclusal através da redução do comprimento da coroa.

Cuidados especiais e monitoramento contínuo também se fazem necessários para o sucesso do tratamento. Controle acurado da higiene bucal, incluindo atenção profissional antes e durante o movimento, é fundamental, visto que a placa supragengival pode contribuir para a formação de placa subgengival, durante a intrusão. Sugerem-se, também, radiografias periapicais periódicas, com intervalos de quatro a seis meses, para monitorar o risco de reabsorções radiculares quando fatores predisponentes forem identificados, como raízes em forma de pipeta ou registro de traumatismos prévios.

Finalmente, vale ressaltar que os mesmos cuidados geralmente tomados quando da utilização da mecânica convencional, em relação à contenção, também devem ser empregados após o movimento de intrusão com a utilização dos miniimplantes. Sugere-se, então, a manutenção destes por um período adicional de cerca de três meses, fazendo-se sua conexão com o dente, ou grupo de dentes que foram movimentados, por meio de um fio de amarrilho, impedindo-se, desta forma, a recidiva do movimento obtido.

Enviado em: julho de 2008 Revisado e aceito: agosto de 2008 


\title{
Orthodontic intrusion with mini-implant anchorage
}

\begin{abstract}
Introduction: Among all different varieties of orthodontically induced tooth movement, intrusion is, without doubt, one of the most difficult movements to be reached. Conventional intrusive biomechanics, although possible, may lead to undesirable side-effects. These events, in most cases, are related to the difficulty in obtaining an efficient anchorage unit. Then, mini-implants, because of their high success rates of effective skeletal anchorage, are valuable devices to orthodontists, transforming the intrusion movement, both at the anterior and the posterior regions, a more and more uncomplicated biomechanical procedure. Aim: to describe and show, clinically, different ways of using mini-implants as an anchorage system to intrusion movement.
\end{abstract}

Key words: Mini-implant. Dental intrusion. Skeletal anchorage.

\section{REFERÊNCIAS}

1. ARAUJO, T. M. Ancoragem esquelética com miniimplantes. In LIMA FILHO, R. M. A.; BOLOGNESE, A. M. Ortodontia: arte e ciência. Maringá: Dental Press, 2007.

2. ARAUJO, T. M.; NASCIMENTO, M. H. A.; BEZERRA, F. SOBRAL, M. C. Ancoragem esquelética em Ortodontia com miniimplantes. Rev. Dental Press Ortodon. Ortop. Facial, Maringá, v. 11, n. 4, p. 126-156, jul./ago. 2006.

3. BAE, S. M.; PARK, H. S.; KYUNG, H. M.; KWON, O. W.; SUNG, J. H. Clinical application of micro-implant anchorage. J. Clin. Orthod., Boulder, v. 36, no. 5, p. 298-302, May 2002.

4. BAE, S. M.; KYUNG, H. M. Mandibular molar intrusion with miniscrew anchorage. J. Clin. Orthod., Boulder, v. 40, no. 2, p. 107-108, Feb. 2006

5. BELINFANTE, L. S.; ABNEY, J. M. A teamwork approach to correct a severe prosthodontic problem. J. Am. Dent. Assoc., Chicago, v. 91, no. 2, p. 357-359, Aug. 1975

6. CARANO, A.; VELO, S.; LEONE, P.; SICILIANI, G. Clinical applications of the miniscrew anchorage system. J. Clin. Orthod., Boulder, v. 39, no. 1, p. 9-24, Jan. 2005.

7. COPE, J. B.; GRAHAM, J. W. Treatment planning for temporary anchorage device applications. In: COPE, J. B. OrthoTADS: the clinical guide atlas. Texas: Under Dog Media, 2007

8. COSTA, A.; RAFFAINL, M.; MELSEN, B. Miniscrews as orthodontic anchorage: a preliminary report. Int. J. Adult. Orthodon. Orthognath. Surg., Chicago, v. 13, no. 3, p. 201-209, 1998.

9. ERVERDI, N.; TOSUN, T.; KELES, A. A new anchorage site for the treatment of anterior open bite: zygomatic anchorage: case report. World J. Orthod., Carol Stream, v. 3, no. 2, p. 147-153, 2002.

10. ERVERDI, N.; USUMEZ, S.; SOLAK, A. New generation openbite treatment with zygomatic anchorage. Angle Orthod. Appleton, v. 76, no. 3, p. 519-526, May 2006.

11. KANOMI, R. Mini-implant for orthodontic anchorage. J. Clin. Orthod., Boulder, v. 31, no. 11, p. 763-767, Nov. 1997

12. KRAVITZ, N. D.; KUSNOTO, B. Posterior impaction with orthodontic miniscrews for openbite closure and improvement of facial profile. World J. Orthod., Carol Stream, v. 8, no. 2 p. 157-166, Sept. 2007.

13. KYUNG, H. M. et al. Handbook for the absoranchor orthodontic micro-implant. 3rd ed. [S.I.: s.n.], 2004.

14. LEE, J. S. Applications of orthodontic mini-implants. 1st ed. Canadá: Quintessence, 2007.

15. LINKOW, L. I. Implanto-orthodontics. J. Clin. Orthod. Boulder, v. 4, no. 12, p. 685-690, Dec. 1970

16. MARASSI, C.; LEAL, A.; HERDY, J. L.; CHIANELLY, O.; SOBREIRA, D. O uso de miniimplantes como auxiliares do tratamento ortodôntico. Ortodontia SPO, São Paulo, v. 38, n. 3, p. 256-265, jul./set. 2005.
17. MASIOLI, D. L. C.; ALMEIDA, M. A. O.; BATITTUCC, E.; MEDEIROS, P. J. Intrusão ortodôntica de molares utilizando mini-placas e parafusos de titânio. Rev. Clin. Ortodon. Dental Press, Maringá, v. 4, n. 5, p. 81-87, out./nov. 2005.

18. MATHEWS, D. P.; KOKICH, V. G. Managing treatment for the orthodontic patient with periodontal problems. Semin.

Orthod., Philadelphia, v. 3, no. 1, p. 21-38, Mar. 1997.

19. PARK, H. S.; KWON, O. W.; SUNG, J. H. Nonextraction treatment of an open bite with microscrew implant anchorage. Am. J. Orthod. Dentofacial Orthop., St. Louis, v. 130, no. 3 p. 391-402, Sept. 2006.

20. PARK, H. S.; KWON, O. W.; SUNG, J. H. Uprighting second molars with micro-implant anchorage. J. Clin. Orthod. Boulder, v. 38, no. 2, p. 100-103, Feb. 2004.

21. POGGIO, P. M.; INCORVATI, C.; VELO, S.; CARANO, A. "Safe zones": a guide for miniscrew positioning in the maxillary and mandibular arch. Angle Orthod., Appleton, v. 76, no. 2, p. 191-197, Mar. 2006.

22. REN, Y.; MALTHA, J. C.; KUIJPERS-JAGTMAN, A. M. Optimum force magnitude for orthodontic tooth movement: a systematic literature review. Angle Orthod., Appleton, v. 73, no. 1, p. 86-92, Feb. 2003

23. ROSEN, P. S.; FORMAN, D. The role of orthognathic surgery in the treatment of severe dentoalveolar extrusion. J. Am. Dent. Assoc., Chicago, v. 130, no. 11, p. 1619-1622, Nov. 1999.

24. SUNG, J. H. et al. Microimplants in Orthodontics. Korea: Dentos, 2006.

25. YAO, C. C.; WU, C. B.; WU, H. Y.; KOK, S. H.; CHANG, H. F.; CHEN, Y. J. Intrusion of the overerupted upper left first and second molars by mini-implants with partial-fixed orthodontic appliances: a case report. Angle Orthod., Appleton, v. 74, no. 4 , p. 550-557, Aug. 2004

\section{Endereço para correspondência}

Telma Martins de Araújo

Av. Araújo Pinho, 62, Centro de Ortodontia e Ortopedia Facial

Prof. José Édimo Soares Martins,

Faculdade de Odontologia da UFBA

CEP: 40.110-150 - Canela - Salvador/BA

E-mail: tmatelma@globo.com 